\title{
Cayley Graphs and Symmetric 4-Polytopes
}

\author{
Barry Monson * \\ University of New Brunswick, Fredericton, New Brunswick, Canada E3B 5A3 \\ Asia Ivić Weiss * \\ York University, Toronto, Ontario, Canada M3J 1P3
}

Received 23 July 2008, accepted 16 November 2008, published online 27 December 2008

\begin{abstract}
Previously we have investigated the medial layer graph $\mathcal{G}$ for a finite, self-dual, regular or chiral abstract 4-polytope $\mathcal{P}$. Here we study the Cayley graph $\mathcal{C}$ on a natural group generated by polarities of $\mathcal{P}$, show that $\mathcal{C}$ covers $\mathcal{G}$ in a readily computable way and construct $\mathcal{C}$ as a voltage graph over $\mathcal{G}$. We then examine such symmetric graphs for several interesting families of polytopes of type $\{p, q, p\}, p=3,4,5$.
\end{abstract}

Keywords: Abstract regular or chiral polytopes, symmetric graphs.

Math. Subj. Class.: 05C25, 51M20

\section{Introduction}

In [7] Coxeter studied the graph on the edges and polygonal faces of each of the two selfdual, regular convex 4-polytopes, then with the second author of this paper, widened the investigation to include certain families of twisted honeycombs [9, 29]. Much later, in [23] and [18], we revisited these objects in the even wider setting of abstract polytopes.

To be specific, we focussed there on finite, self-dual, abstract regular (or chiral) 4-polytopes $\mathcal{P}$. In fact, self-duality served mainly to help guarantee vertex-transitivity in the associated graph $\mathcal{G}$. Here we take a closer look at the full group $D$ of dualities and automorphisms for $\mathcal{P}$. We are particularly interested in the Cayley graph $\mathcal{C}$ on the subgroup of $D$ generated by a natural set of polarities (dualities of period 2); see Definition 3 and the remarks after it. In Proposition 11 we show that $\mathcal{C}$ is symmetric, indeed transitive on $t$-arcs for some $t \geq 1$. Proposition 12 then describes how $\mathcal{C}$ covers the edge-face graph $\mathcal{G}$ mentioned earlier; also, in many cases $\mathcal{C}$ can be naturally constructed as a voltage graph over $\mathcal{G}$ (Proposition 14). Having established this foundation, we then take a detailed look at several interesting classes of polytopes. Before proceeding, however, we want to thank Marston Conder, Tomo Pisanski, Egon Schulte and the referees for several helpful comments.

\footnotetext{
* Supported by the NSERC of Canada.

E-mail addresses: bmonson@unb.ca (Barry Monson),weiss@mathstat.yorku.ca (Asia Ivić Weiss)
} 


\section{Polytopes and graphs}

Rather than repeat in detail what can be found elsewhere, we refer the reader to [23] and [18] for notation and general background, and to [2] and [17] for a deeper discussion of symmetric graphs and polytopes. Here let us simply recall that an abstract $n$-polytope $\mathcal{P}$ is a partially ordered set having some of the key combinatorial properties of the face lattice of a convex $n$-polytope; in general, however, $\mathcal{P}$ need not be a lattice, need not be finite, need not have any familiar geometric realization.

Beginning with any abstract 4-polytope $\mathcal{P}$, one can construct a simple, bipartite graph $\mathcal{G}=\mathcal{G}(\mathcal{P})$, whose nodes are all 1 -faces (edges) and 2 -faces (polygons) in $\mathcal{P}$, where two such nodes are joined by a branch when incident as faces of $\mathcal{P}$. (We employ the lesser-used terms 'node' and 'branch' since we occasionally discuss the edges and vertices ( 0 -faces) of $\mathcal{P}$ itself.) Following [23, 18] we say that $\mathcal{G}$ is the medial layer graph of $\mathcal{P}$. Viewing $\mathcal{P}$ as a ranked, partially ordered set, then $\mathcal{G}$ is quite literally the graph on the elements of rank 1 or 2 in a Hasse diagram for $\mathcal{P}$.

Usually $\mathcal{P}$ will be equivelar, say with Schläfli type $\{p, q, r\}$. This encodes a sort of local symmetry in which every 2 -face of $\mathcal{P}$ is a $p$-gon, with $r$ such $p$-gons around every edge; furthermore, in each facet (3-face) of $\mathcal{P}$ there are $q$ edges (and hence $q$ polygons) around each vertex. Consequently, the graph $\mathcal{G}$ contains cycles of length $2 q$ and has bipartition classes consisting, respectively, of $p$-valent and $r$-valent nodes.

In fact, we shall enforce equivelarity by demanding that the group $A=A(\mathcal{P})$ of order preserving bijections (automorphisms) on $\mathcal{P}$ be quite large. To frame our discussion we fix a base flag $\Phi=\left\{F_{0}, F_{1}, F_{2}, F_{3}\right\}$ (suppressing the improper faces $F_{-1}, F_{4}$ ). For $0 \leq j \leq 3$, there is a unique $j$-adjacent flag $\Phi^{j}$ which differs from $\Phi$ in just the proper face $F_{j}$.

Now recall from [17] that $\mathcal{P}$ is regular if $A$ is transitive on flags. For $0 \leq j \leq 3$, let $\rho_{j}$ be the unique automorphism mapping $\Phi$ to $\Phi^{j}$. Then $A$ is generated by the involutions $\rho_{j}$, which we usefully think of as 'reflections' (see [17, 2B]); moreover, $\mathcal{P}$ is equivelar of type $\{p, q, r\}$, where $p, q, r$ are the periods of the 'rotations' $\sigma_{1}:=\rho_{0} \rho_{1}, \sigma_{2}:=\rho_{1} \rho_{2}$ and $\sigma_{3}:=\rho_{2} \rho_{3}$, respectively. The rotation group

$$
A^{+}:=\left\langle\sigma_{1}, \sigma_{2}, \sigma_{3}\right\rangle
$$

has index at most 2 in $A$. If this index equals 2 we say that $\mathcal{P}$ is directly regular. Otherwise, $A^{+}=A$, and we say that $\mathcal{P}$ is non-orientably regular (since the order complex for $\mathcal{P}$ must be non-orientable; see [17, 2C] and [26, p. 501]).

Relaxing symmetry a little, we say that $\mathcal{P}$ is chiral if $A$ has just two flag orbits, with adjacent flags always in different orbits [26]. Here the automorphism group is also generated by 'rotations' behaving much like the $\sigma_{j}$ in the directly regular case. In this case, too, we have $A=A^{+}$; and again $\mathcal{P}$ is equivelar of type $\{p, q, r\}$.

If $\mathcal{P}$ is regular or chiral, the generating rotations $\sigma_{i}$ satisfy the standard relations

$$
\sigma_{1}^{p}=\sigma_{2}^{q}=\sigma_{3}^{r}=\left(\sigma_{1} \sigma_{2}\right)^{2}=\left(\sigma_{2} \sigma_{3}\right)^{2}=\left(\sigma_{1} \sigma_{2} \sigma_{3}\right)^{2}=1,
$$

(and usually other independent relations). Moreover, apart from one special case, we have the following intersection condition on special subgroups:

$$
\begin{array}{ccc}
\left\langle\sigma_{1}\right\rangle \cap\left\langle\sigma_{2}\right\rangle & = & \{1\} \\
\left\langle\sigma_{2}\right\rangle \cap\left\langle\sigma_{3}\right\rangle & = & \{1\} \\
\left\langle\sigma_{1}, \sigma_{2}\right\rangle \cap\left\langle\sigma_{2}, \sigma_{3}\right\rangle & = & \left\langle\sigma_{2}\right\rangle .
\end{array}
$$


However, if $\mathcal{P}$ is non-orientably regular, the very last intersection sometimes becomes

$$
\left\langle\sigma_{1}, \sigma_{2}\right\rangle \cap\left\langle\sigma_{2}, \sigma_{3}\right\rangle=\left\langle\rho_{1}, \rho_{2}\right\rangle .
$$

(For regular polytopes, the subgroup $\left\langle\sigma_{2}\right\rangle$ always has index 2 in $\left\langle\rho_{1}, \rho_{2}\right\rangle$.) One particularly nice instance of this is the 11-cell described in Example 17 below.

Remarks. When $\mathcal{P}$ is a regular 4-polytope, so that $A=\left\langle\rho_{0}, \rho_{1}, \rho_{2}, \rho_{3}\right\rangle$, the equations in (3) are related to the perhaps more familiar result that

$$
\left\langle\rho_{i}: i \in I\right\rangle \cap\left\langle\rho_{i}: i \in J\right\rangle=\left\langle\rho_{i}: i \in I \cap J\right\rangle,
$$

for any subsets $I, J \subseteq\{0,1,2,3\}$. This in turn recalls a well-known fact concerning parabolic subgroups of general Coxeter groups [15, Theorem 5.5(c)]. Now in either regular or chiral cases, it is possible to reconstruct $\mathcal{P}$ in a natural way as a coset geometry over $A$ ([17, $2 \mathrm{E}]$ and $[26, \S 5])$. Conditions (3) or (4) translate in this construction into essential properties of $\mathcal{P}$; for example, in a 4 -polytope every 2 -face will be incident with exactly two 3 -faces.

Lemma 1. $\sigma_{2}^{2}=\sigma_{1}^{-1} \sigma_{3} \sigma_{1} \sigma_{3}^{-1} \in\left\langle\sigma_{1}, \sigma_{3}\right\rangle$.

Proof. From (2) we have

$$
\begin{aligned}
\sigma_{2}^{2} & =\sigma_{1}^{-1} \sigma_{1} \sigma_{2}^{2} \\
& =\sigma_{1}^{-1} \sigma_{1} \sigma_{2}\left(\sigma_{1} \sigma_{2} \sigma_{3}\right)^{2} \sigma_{2} \\
& =\sigma_{1}^{-1}\left(\sigma_{1} \sigma_{2}\right)^{2} \sigma_{3} \sigma_{1}\left(\sigma_{2} \sigma_{3} \sigma_{2}\right) \\
& =\sigma_{1}^{-1} \sigma_{3} \sigma_{1} \sigma_{3}^{-1} .
\end{aligned}
$$

Corollary 2. If $q$ is odd, then $\sigma_{2}=\left(\sigma_{3} \sigma_{1}^{-1} \sigma_{3}^{-1} \sigma_{1}\right)^{\frac{q-1}{2}} \in\left\langle\sigma_{1}, \sigma_{3}\right\rangle$.

Clearly the graph $\mathcal{G}$ inherits considerable symmetry from the polytope $\mathcal{P}$. If $\mathcal{P}$ is regular or chiral, then $A$ acts transitively on each of the two sets of 1 -arcs in $\mathcal{G}$, namely those with initial node a 1 -face or 2 -face of $\mathcal{P}$.

Of course, no automorphism of $\mathcal{P}$ maps a 1 -face to a 2 -face, so that $A$ cannot be transitive on the set of all nodes of $\mathcal{G}$. As a remedy, we may also suppose $\mathcal{P}$ to be self-dual. In this case, $A=A(\mathcal{P})$ has index 2 in the group $D=D(\mathcal{P})$ of all dualities and automorphisms. Furthermore, the graph $\mathcal{G}$ is symmetric, that is, $\operatorname{Aut}(\mathcal{G})$ is transitive on the set of all 1 -arcs $[2$, p. 118]. We refer to $[23,18]$ for a discussion of such graphs in the case that $p=r=3$.

Elaborating on [14, p. 131], we say that $\mathcal{P}$ is properly self-dual if there exists a duality preserving each flag orbit (under the natural action of $A$ ). This is forced when $\mathcal{P}$ is regular but can fail in chiral cases. Indeed, an improperly self-dual chiral polytope admits dualities which swap the two flag orbits [14, Lemma 3.1]. Whenever $\mathcal{P}$ is self-dual and regular or properly self-dual and chiral, there must exist a polarity $\delta$ which flips the base flag, that is, transposes $F_{0}, F_{3}$ and $F_{1}, F_{2}$. This implies that

and that $p=r$.

$$
\begin{array}{ccc}
\delta^{2} & = & 1 \\
\delta^{-1} \sigma_{1} \delta & = & \sigma_{3}^{-1} \\
\delta^{-1} \sigma_{2} \delta & = & \sigma_{2}^{-1}
\end{array}
$$

Conventions. We henceforth assume that the polytope $\mathcal{P}$ is self-dual and regular or properly self-dual and chiral, with Schläfli type $\{p, q, p\}$, where $p<\infty$. (We allow $q=\infty$.) For convenience below, we take 'self-dual' to mean 'properly self-dual' in chiral cases. 


\section{A group generated by polarities}

Now let us consider the group $D^{+}:=\left\langle\sigma_{1}, \sigma_{2}, \sigma_{3}, \delta\right\rangle$ of all rotations and dualities, which by (5) splits as

$$
D^{+} \simeq A^{+} \rtimes C_{2},
$$

with $C_{2}=\langle\delta\rangle$. Also, we define, in symmetrical fashion,

$$
\delta_{j}=\sigma_{1}^{1-j} \delta \sigma_{1}^{j-1}, \text { for } 1 \leq j \leq p,
$$

so that $\delta_{1}=\delta$. Now we may define the group of most interest to us here:

Definition 3. Let $G=\left\langle\delta_{1}, \ldots, \delta_{p}\right\rangle$ be the subgroup of $D^{+}$defined by the above set of distinguished polarities.

Observe that these generating polarities are distinct: if say $\delta_{1}=\delta_{j}$, then

$$
F_{2} \delta=F_{2} \sigma_{1}^{1-j} \delta \sigma_{1}^{j-1}
$$

so that $F_{1}=F_{1} \sigma_{1}^{j-1}$, which forces $j \equiv 1 \bmod p$.

Remarks. By working within the group $D^{+}$(rather than all of $D$ ), we can readily tackle both regular and chiral polytopes in one go. Our choice of the polarities $\delta_{j}$ is natural for the study of local properties of the medial layer graph $\mathcal{G}$, since the $\delta_{j}$ 's map a base vertex of $\mathcal{G}$ to its neighbours. Looking ahead to Proposition 9, we note as well that the group $G$ can be as large as $D^{+}$, so it is hard to see how another set of generators could be more versatile. See also the comments after Definition 10.

Anticipating the subgroups described in (7) and (8) below, we now have the following arrangement of groups:

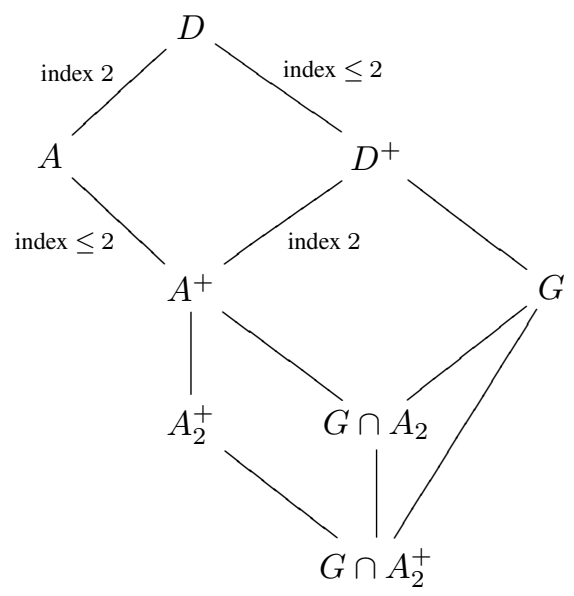

Figure 1: Duality and automorphism groups for self-dual regular or chiral polytopes.

Our ultimate goal is to understand a Cayley graph on the group $G$ (see Definition 10); but first we must examine $G$ more carefully. 
Lemma 4. Taking subscripts modulo $p$, we have
(a) $\delta_{j+k}=\sigma_{1}^{-k} \delta_{j} \sigma_{1}^{k}$;
(b) $\delta_{j} \delta_{k}=\sigma_{1}^{1-j} \sigma_{3}^{k-j} \sigma_{1}^{k-1}$; in particular, $\delta_{1} \delta_{2}=\sigma_{3} \sigma_{1}$ and $\delta_{p} \delta_{1}=\sigma_{1} \sigma_{3}$;
(c) $\sigma_{2}^{2}=\delta_{2} \delta_{3} \delta_{2} \delta_{1}$;
(d) $\sigma_{3} \delta_{j} \sigma_{3}^{-1}=\delta_{1} \delta_{2} \delta_{j+1} \delta_{2} \delta_{1}$;
(e) $\left(\sigma_{2} \sigma_{3}\right)^{-1} \delta_{j} \sigma_{2} \sigma_{3}=\delta_{3-j}$;
(f) $\sigma_{2}^{-1} \delta_{j} \sigma_{2}=\delta_{1} \delta_{2} \delta_{4-j} \delta_{2} \delta_{1}$ and $\sigma_{2} \delta_{j} \sigma_{2}^{-1}=\delta_{2} \delta_{3} \delta_{4-j} \delta_{3} \delta_{2}$.

Proof. Part (a) is routine. For (b) note that

$$
\delta_{j} \delta_{k}=\sigma_{1}^{1-j} \delta \sigma_{1}^{j-1} \sigma_{1}^{1-k} \delta \sigma_{1}^{k-1}=\sigma_{1}^{1-j} \sigma_{3}^{k-j} \sigma_{1}^{k-1} .
$$

Part (c) follows from (b) and Lemma 1. From (b) we also have $\sigma_{3}=\delta_{1} \delta_{2} \sigma_{1}^{-1}$, so that

$$
\sigma_{3} \delta_{j} \sigma_{3}^{-1}=\delta_{1} \delta_{2} \sigma_{1}^{-1} \delta_{j} \sigma_{1} \delta_{2} \delta_{1}=\delta_{1} \delta_{2} \delta_{j+1} \delta_{2} \delta_{1}
$$

For part (e) we use (2), which gives $\sigma_{1}^{-1}=\sigma_{2} \sigma_{3} \sigma_{1} \sigma_{2} \sigma_{3}$, so that $\left(\sigma_{2} \sigma_{3}\right) \sigma_{1}^{j}=\sigma_{1}^{-j}\left(\sigma_{2} \sigma_{3}\right)$, for $1 \leq j \leq p$. Thus

$$
\begin{aligned}
\left(\sigma_{2} \sigma_{3}\right)^{-1} \delta_{j}\left(\sigma_{2} \sigma_{3}\right) & =\sigma_{2} \sigma_{3} \sigma_{1}^{1-j} \delta \sigma_{1}^{j-1} \sigma_{2} \sigma_{3} \\
& =\sigma_{1}^{j-1} \sigma_{2} \sigma_{3} \delta \sigma_{2} \sigma_{3} \delta \delta \sigma_{1}^{1-j} \\
& =\sigma_{1}^{j-1} \sigma_{2} \sigma_{3} \sigma_{2}^{-1} \sigma_{1}^{-1} \delta \sigma_{1}^{1-j} \\
& =\sigma_{1}^{j-1} \sigma_{2} \sigma_{3} \sigma_{1} \sigma_{2} \delta \sigma_{1}^{1-j} \\
& =\sigma_{1}^{j-1} \sigma_{1}^{-1} \sigma_{3}^{-1} \delta \sigma_{1}^{1-j} \\
& =\sigma_{1}^{j-2} \delta \sigma_{1}^{2-j} \\
& =\delta_{3-j} .
\end{aligned}
$$

Finally, part (f) follows at once from (c), (d) and (e).

Remarks. When $p=2$, the polytope $\mathcal{P}=\{2, q, 2\}$ is uniquely specified by its Schläfli symbol. Both $\rho_{0}$ and $\rho_{3}$ act trivially on the graph $\mathcal{G}$, which is then a $2 q$-cycle.

However, as we observed in [23, $\S 2], D$ does act faithfully on $\mathcal{G}$ when $p(=r) \geq 3$. In this case, we can regard $D$ as a subgroup of $\operatorname{Aut}(\mathcal{G})$. A fragment of a polytope of type $\{3, q, 3\}$ is displayed in Figure 2. The nodes of the graph $\mathcal{G}$ are indicated by black and white discs; and $\delta_{1}, \delta_{2}, \delta_{3}$ swap the (black and white) nodes incident with the nearby branches.

It is useful to recall here that, in chiral and directly regular cases, the stabilizer in $A^{+}$of the node $v_{2}=F_{2}$ (the base 2-face of $\mathcal{P}$ ) is the subgroup

$$
A_{2}^{+}:=\left\langle\sigma_{1}, \sigma_{2} \sigma_{3}\right\rangle \text {, }
$$

which is dihedral of order $2 p$. In the non-orientably regular case, $A=A^{+}$and the stabilizer of $F_{2}$ is now

$$
A_{2}:=\left\langle\rho_{0}, \rho_{1}, \rho_{3}\right\rangle
$$

which has order $4 p$. Note that the index $\left[A_{2}: A_{2}^{+}\right]=2$. In Figure $2, \sigma_{1}$ acts on $F_{2}$ (here a triangle) like a 'rotation' ( $\left.v_{1} \rightarrow v_{3} \rightarrow w_{2} \rightarrow v_{1}\right)$, whereas $\sigma_{2} \sigma_{3}$ acts like a 'reflection' $\left(v_{1} \leftrightarrow v_{3}, w_{2} \leftrightarrow w_{2}\right)$. 


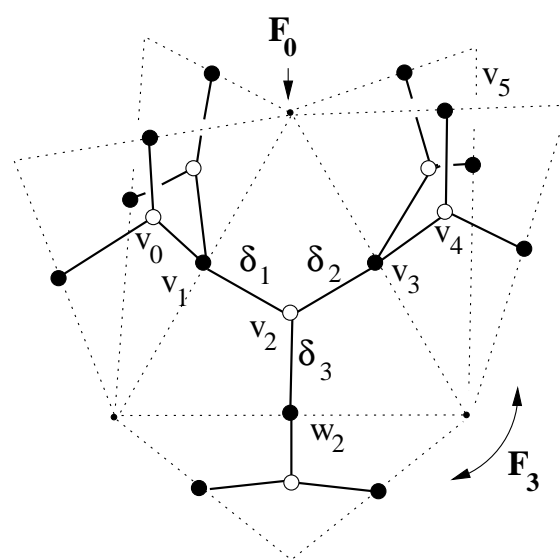

\section{Base Flag}

$\mathbf{F}_{0}, \mathbf{F}_{1}=\mathbf{v}_{1}, \mathbf{F}_{2}=\mathbf{v}_{2}, \mathbf{F}_{3}$

Figure 2: A fragment of a polytope of type $\{3, q, 3\}$.

Corollary 5. G is a normal subgroup of $D^{+}$.

Proof. This follows at once from Lemma 4 (a),(d),(f).

We can now enumerate the cosets of $G$ in $D^{+}$, though perhaps with some redundancy. Indeed, we define cosets

$$
a_{j}:=G \sigma_{1}^{j-1} ; \quad b_{j}:=a_{j} \sigma_{2}, \text { for } 1 \leq j \leq p .
$$

(Recall that we assume $p<\infty$.)

Lemma 6. Again taking subscripts modulo $p$, we have

\begin{tabular}{l|l|l|l|l}
$a_{j} \sigma_{1}=a_{j+1}$ & $a_{j} \sigma_{2}=b_{j}$ & $a_{j} \sigma_{2}^{-1}=b_{j}$ & $a_{j} \sigma_{3}=a_{j-1}$ & $a_{j} \delta=a_{j}$ \\
\hline$b_{j} \sigma_{1}=b_{j-1}$ & $b_{j} \sigma_{2}=a_{j}$ & $b_{j} \sigma_{2}^{-1}=a_{j}$ & $b_{j} \sigma_{3}=b_{j+1}$ & $b_{j} \delta=b_{j}$ \\
\hline
\end{tabular}

Moreover, all cosets of $G$ in $D^{+}$occur among the $a_{j}, b_{j}, 1 \leq j \leq p$.

Proof. These identitites follow easily from Lemma 4 and Corollary 5. For example,

$$
b_{j} \sigma_{2}=G \sigma_{1}^{j-1} \sigma_{2}^{2}=\sigma_{1}^{j-1} \sigma_{2}^{2} G=\sigma_{1}^{j-1} G=G \sigma_{1}^{j-1}=a_{j} .
$$

Since $a_{1}=G$, every coset of $G$ in $D^{+}$is counted among the $a_{j}, b_{j}$.

Proposition 7. $G$ acts transitively on the nodes of $\mathcal{G}$.

Proof. $D^{+}$certainly acts transitively on nodes of $\mathcal{G}$. But from Lemma 6 we find that every coset of $G$ in $D^{+}$has a representative in $A_{2}^{+}$(see (7)). Indeed, $\sigma_{1}^{j-1} \in A_{2}^{+}$, so we need only observe that $G \sigma_{1}^{j-2} \sigma_{2} \sigma_{3}=a_{j-1} \sigma_{2} \sigma_{3}=b_{j-1} \sigma_{3}=b_{j}$. It follows that $G$ acts transitively on nodes of $\mathcal{G}$. 
Keeping in place a fixed regular or chiral, but self-dual polytope $\mathcal{P}$ with Schläfli symbol $\{p, q, p\}$, we now introduce the universal polytope $\mathcal{U}$ of type $\{p, \infty, p\}$. Certainly, $\mathcal{U}$ is directly regular and self-dual; and its automorphism group $\mathrm{A}(\mathcal{U})$ is the Coxeter group $[p, \infty, p]$ with diagram

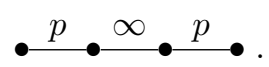

(In fact, we could replace the ' $\infty$ ' by any even multiple of $q$.) For ease of notation, we let $\bar{A}, \bar{D}^{+}$and so on denote objects attached to $\mathcal{U}$. In particular,

$$
\bar{D}^{+}=\left\langle\bar{\sigma}_{1}, \bar{\sigma}_{2}, \bar{\sigma}_{3}, \bar{\delta}\right\rangle .
$$

We note that defining relations for $\bar{D}^{+}$are obtained by setting $p=r, q=\infty$ in (2) and (5) (and replacing $\sigma_{j}$ by $\bar{\sigma}_{j}, \delta$ by $\bar{\delta}$ ).

Clearly the mapping $\bar{\sigma}_{j} \mapsto \sigma_{j}, \bar{\delta} \mapsto \delta$ induces an epimorphism $g: \bar{D}^{+} \rightarrow D^{+}$. Thus $\bar{K}:=\operatorname{ker}(g)$ is generated, as a normal subgroup of $\bar{D}^{+}$, by any relations $R$ which we must adjoin to (2) in order to establish a presentation for $D^{+}$.

Even if the cosets in Lemma 6 are redundant, the action described there does suggest that we also investigate the dihedral group

$$
I_{2}(p)=\left\langle\alpha_{0}, \alpha_{1} \mid \alpha_{0}^{2}=\alpha_{1}^{2}=\left(\alpha_{0} \alpha_{1}\right)^{p}=1\right\rangle,
$$

of order $2 p$. It is easy to check that the mapping

$$
\bar{\sigma}_{1} \mapsto \alpha_{0} \alpha_{1}, \bar{\sigma}_{2} \mapsto \alpha_{0}, \bar{\sigma}_{3} \mapsto \alpha_{1} \alpha_{0}, \bar{\delta} \mapsto 1
$$

induces another epimorphism $f: \bar{D}^{+} \rightarrow I_{2}(p)$. Clearly, $\bar{G} \subseteq \operatorname{ker}(f)$. But $\bar{G}$ has index at most $2 p$ in $\bar{D}^{+}$, by Lemma 6 . Thus $\bar{G}=\operatorname{ker}(f)$, and the index equals $2 p$ in the universal setting. The situation is summarized in Figure 3.

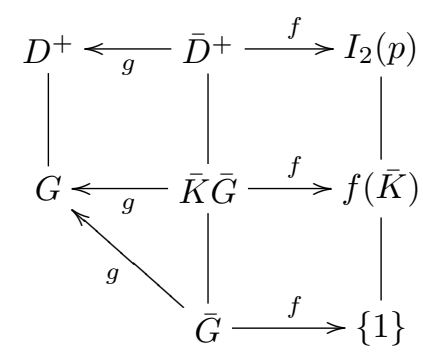

Figure 3: The index of $G$ in $D^{+}$.

Returning to the polytope $\mathcal{P}$, we can now discuss a useful covering parameter. We let $k=$ $k(\mathcal{P})$ denote the order of the subgroup $G \cap A_{2}^{+}$(see Figure 1).

Lemma 8. Let $f(\bar{K})$ be the normal subgroup of $I_{2}(p)$ generated by the images $f(R)$ of the 'additional' relations required in a presentation of $D^{+}$. Then

$$
I_{2}(p) / f(\bar{K}) \simeq D^{+} / G \simeq A_{2}^{+} /\left(G \cap A_{2}^{+}\right) .
$$

Furthermore, $k=|f(\bar{K})|$ and the index of $G$ in $D^{+}$equals $2 p / k$. Also, in non-orientably regular cases, $\left|G \cap A_{2}\right|=2 k$. 
Proof. Since $\bar{K} \bar{G} \triangleleft \bar{D}^{+}$, we immediately get $I_{2}(p) / f(\bar{K}) \simeq D^{+} / G$. We easily check that $G A_{2}^{+}=D^{+}$, so $D^{+} / G \simeq A_{2}^{+} /\left(G \cap A_{2}^{+}\right)$follows from the second isomorphism theorem. But $A_{2}^{+} \simeq I_{2}(p)$, so that $k=\left|G \cap A_{2}^{+}\right|=|f(\bar{K})|$.

When $\mathcal{P}$ is non-orientably regular, we have $A=A^{+}$, so that $D=D^{+}=G A_{2}^{+}=G A_{2}$. Thus, $G \cap A_{2}$ also has index $2 p / k$ in $A_{2}$. Since $\left|A_{2}\right|=4 p$, we have $\left|G \cap A_{2}\right|=2 k$.

Now suppose, for example, that $q$ is odd, so that $f(\bar{K})$ contains $f\left(\bar{\sigma}_{2}^{q}\right)=\alpha_{0}^{q}=\alpha_{0}$. Thus $f(\bar{K})$ contains the dihedral subgroup generated by the conjugacy class of the reflection $\alpha_{0}$ in $I_{2}(p)$. Hence $f(\bar{K})$ is the full group $I_{2}(p)$ if $p$ is odd as well, but could have index 2 when $p$ is even. We have verified

Proposition 9. Suppose $\mathcal{P}$ is self-dual and regular or chiral of type $\{p, q, p\}$, with $q$ odd. If $p$ is odd, then $G=D^{+}$. If $p$ is even, then $G$ has index at most 2 in $D^{+}$.

At last we are ready for the following

Definition 10. Let $\mathcal{C}=\mathcal{C}(\mathcal{P})$ be the Cayley graph constructed on the group $G$, with specified (involutory) generators $\delta_{1}, \ldots, \delta_{p}$.

Remarks. Recall from [2, ch. 16] that $\mathcal{C}$ has node set $G$, where $\mu, \nu \in G$ are adjacent if and only if $\mu \nu^{-1}=\delta_{j}$ for some $j \in\{1, \ldots, p\}$. Since all $\delta_{j}^{2}=1$, this unambiguously defines $\mathcal{C}$ as a simple $p$-valent graph.

Since $\mu \tau(\nu \tau)^{-1}=\mu \nu^{-1}$, for all $\tau \in G$, the right regular action of $G$ on itself serves to embed $G$ as a subgroup of $\operatorname{Aut}(\mathcal{C})$. Similarly, if $\varphi \in \operatorname{Aut}(G)$ globally fixes the set $\left\{\delta_{1}, \ldots, \delta_{p}\right\}$, then $\varphi$ induces a automorphism of $\mathcal{C}$ which stabilizes the vertex 1 .

Such considerations also point out the unsuitability of other polarities, such as the $\sigma_{2}^{1-j} \delta$ $\sigma_{2}^{j-1}, 1 \leq j \leq q$, as possible generators. For one thing, the corresponding Cayley graph will have no obvious local symmetry, because of Lemma 4(f).

Let us look more closely at our graph $\mathcal{C}$. It is clear on geometrical grounds, and follows easily from Lemma 4(a), (e), that the dihedral group $A_{2}^{+}=\left\langle\sigma_{1}, \sigma_{2} \sigma_{3}\right\rangle$ normalizes $\left\{\delta_{1}, \ldots, \delta_{p}\right\}$, and, in fact, acts transitively and faithfully on that set, just as it would act on the vertices of a $p$-gon. This gives

Proposition 11. The Cayley graph $\mathcal{C}$ is a connected, symmetric, p-valent graph. The node stabilizer in Aut $(\mathcal{C})$ contains a dihedral subgroup of order $2 p$.

Remarks. From Proposition 9 we note that $A_{2}^{+} \subset G$ is possible. Thus we cannot generally hope that $\operatorname{Aut}(\mathcal{C})$ contains a subgroup isomorphic to $G \rtimes A_{2}^{+}$.

We also note that in non-orientably regular cases, it can never happen that the full $F_{2^{-}}$ stabilizer $A_{2}=\left\langle\rho_{0}, \rho_{1}, \rho_{3}\right\rangle$ normalizes $\left\{\delta_{1}, \ldots, \delta_{p}\right\}$. For example, suppose $\rho_{0} \delta_{1} \rho_{0}=\delta_{j}$. Then

$$
F_{1}=F_{2} \rho_{0} \delta_{1} \rho_{0}=F_{2} \delta_{j}=F_{1} \sigma_{1}^{j-1},
$$

so that $j=1$. But then $F_{0}=F_{3} \rho_{0} \delta_{1}=F_{3} \delta_{1} \rho_{0}=F_{0} \rho_{0}$, a contradiction.

Proposition 12. There is a surjective graph homomorphism

$$
h: \mathcal{C} \rightarrow \mathcal{G},
$$


which is injective on any neighbourhood (of nodes distant at most 1 from a given node). Moreover, if $\mathcal{P}$ is chiral or directly regular, then $\mathcal{C}$ is a $k$-fold covering of $\mathcal{G}$, where $k=$ $\left|G \cap A_{2}^{+}\right|=|f(\bar{K})|$. If $\mathcal{P}$ is non-orientably regular, then $\mathcal{C}$ is a $2 k$-fold covering of $\mathcal{G}$.

Proof. We let $h: \mu \rightarrow\left(F_{2}\right) \mu$, for all $\mu \in G$. Note that $\mu$ is a typical node of $\mathcal{C}$; and $h$ is surjective by Proposition 7. Now suppose that $\mu, \nu \in G$ are adjacent nodes with $\mu \nu^{-1}=\delta_{j}$. Then

$$
F_{2} \mu=F_{2} \delta_{j} \nu=F_{1} \sigma_{1}^{j-1} \nu
$$

is incident with $F_{2} \nu$, since $F_{1} \sigma_{1}^{j-1}$ is a 1 -face of $F_{2}$. Thus $h$ is a graph homomorphism. The $p$ neighbours of $\mu$ in $\mathcal{C}$ are the nodes $\delta_{1} \mu, \ldots, \delta_{p} \mu$. Their images under $h$ are distinct since $\mu$ acts bijectively on $\mathcal{P}$.

Finally, we note that a typical node $F_{2} \mu$ of $\mathcal{G}$ is covered by $\lambda \in G$ if and only if $\lambda \mu^{-1} \in$ $\operatorname{Stab}_{G}\left(F_{2}\right)=G \cap \operatorname{Stab}_{A^{+}}\left(F_{2}\right)$. But in chiral or directly regular cases, the latter group is just $G \cap A_{2}^{+}$and has order $k$. The non-orientably regular case also follows from Lemma 8 .

At least in chiral and directly regular cases, we can exploit the foregoing proof to describe $\mathcal{C}$ as a derived voltage graph over the base graph $\mathcal{G}$, with voltage group $S:=\operatorname{Stab}_{G}\left(F_{2}\right)=$ $G \cap A_{2}^{+}$(see [12, Ch. 2] or [2, Ch. 19]). Recall that we must first assign to each 1-arc [u,v] in $\mathcal{G}$ a voltage $\varphi[u, v] \in S$, satisfying $\varphi[v, u]=(\varphi[u, v])^{-1}$.

Lemma 13. Suppose $\alpha \delta_{i}=\beta \delta_{j}$, for $\alpha, \beta \in S$ and $1 \leq i, j \leq p$. Then $i=j$ and $\alpha=\beta$.

Proof. Since $F_{2} \sigma_{1}=F_{2}$, we have $F_{2} \alpha \sigma_{1}^{1-i} \delta \sigma_{1}^{i-1}=F_{2} \beta \sigma_{1}^{1-j} \delta \sigma_{1}^{j-1}$, so $F_{1} \sigma_{1}^{i-1}=F_{1} \sigma_{1}^{j-1}$. Thus $i=j$.

By Proposition 7, the nodes of $\mathcal{G}$ correspond to a (right) transversal $\left\{\mu_{1}, \ldots, \mu_{e}\right\}$ for the subgroup $S$ in $G$. Thus, for a typical 1 -arc $[u, v]$ in $\mathcal{G}$ we may take $u=F_{2} \mu_{x}$ and $v=F_{2} \mu_{y}=F_{2} \delta_{j} \mu_{x}$ for uniquely determined $x, y \in\{1, \ldots, e\}$ and $j \in\{1, \ldots, p\}$. We define

$$
\varphi[u, v]:=\delta_{j} \mu_{x} \mu_{y}^{-1} .
$$

Note that $\varphi[u, v] \in S$, and

$$
\mu_{y} \mu_{x}^{-1}=(\varphi[u, v])^{-1} \delta_{j} \varphi[u, v](\varphi[u, v])^{-1}=\delta_{i}(\varphi[u, v])^{-1},
$$

say, since $S$ normalizes $\left\{\delta_{1}, \ldots, \delta_{p}\right\}$. (See the remarks after Proposition 11.)

Since $\delta_{i} \mu_{y} \mu_{x}^{-1} \in S$, we conclude from Lemma 13 that $i=j$ and $\varphi[v, u]=(\varphi[u, v])^{-1}$.

Proposition 14. Suppose $\mathcal{P}$ is chiral or directly regular. Then the Cayley graph $\mathcal{C}$ is isomorphic to the derived voltage graph $\mathcal{G}^{\varphi}$.

Proof. Recall ([2, Ch. 19]) that the nodes of $\mathcal{G}^{\varphi}$ are all pairs $(\gamma, u)$, where $u$ is a node of $\mathcal{G}$ and $\gamma \in S$, with $(\gamma, u) \sim(\lambda, v)$ if and only if $u \sim v$ in $\mathcal{G}$ and $\lambda=\gamma \cdot \varphi[u, v]$. But each node of $\mathcal{C}$ can be uniquely written as $\gamma \mu_{x}$, where $1 \leq x \leq e$ and $\gamma \in S$. We may therefore define a map

$$
\begin{aligned}
F: \mathcal{C} & \rightarrow \mathcal{G}^{\varphi} \\
\gamma \mu_{x} & \mapsto\left(\gamma, F_{2} \mu_{x}\right)
\end{aligned}
$$

Clearly $F$ is a bijection. Consider two adjacent nodes in $\mathcal{C}$, say $\mu=\gamma \mu_{x}, \nu=\lambda \mu_{y}$, where $\gamma, \lambda \in S$ and $\mu \nu^{-1}=\delta_{j}$. Again since $S$ normalizes $\left\{\delta_{1}, \ldots, \delta_{p}\right\}$, we have $\gamma^{-1} \delta_{j} \gamma=\delta_{i}$ and

$$
\mu_{y}=\lambda^{-1} \delta_{j} \gamma \mu_{x}=\left(\lambda^{-1} \gamma\right) \delta_{i} \mu_{x}
$$


Thus $F_{2} \mu_{y}=F_{2} \delta_{i} \mu_{x}$, so $F_{2} \mu_{y}$ and $F_{2} \mu_{x}$ are adjacent in $\mathcal{G}$ and

$$
\gamma \cdot \varphi\left[F_{2} \mu_{x}, F_{2} \mu_{y}\right]=\gamma \delta_{i} \mu_{x} \mu_{y}^{-1}=\lambda .
$$

Thus $F$ preserves adjacency (as does its inverse).

\section{Finite polytopes of type $\{3, q, 3\}$}

Taking $p=3$ in the more general discussion of the previous section, we find that both $\mathcal{C}$ and $\mathcal{G}$ are symmetric trivalent graphs. Much more is known about this very interesting family of graphs, both in general $([1,2,3,5,6])$ and in the specific case of medial layer graphs for regular or chiral polytopes $([23,18])$. Although we could generalize a little, we shall assume that $\mathcal{P}$ is finite, so that the graphs $\mathcal{C}$ and $\mathcal{G}$ are finite, too.

From Propositions 9, 11 and 12, we immediately get

Proposition 15. Suppose $\mathcal{P}$ is finite, self-dual and regular or chiral of type $\{3, q, 3\}$, with $q$ odd. Then $D^{+}=G=\left\langle\delta_{1}, \delta_{2}, \delta_{3}\right\rangle$, whose generators satisfy at least the relations implicit in the diagram

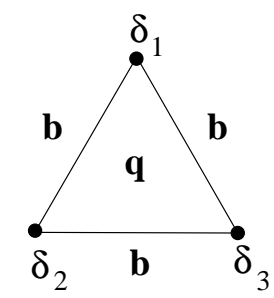

in which $b$ is the order of the (left) Petrie motion $\sigma_{1} \sigma_{3}$ for $\mathcal{P}$, and $q$ is the order of $\sigma_{2}$ (and of $\left.\delta_{1} \delta_{2} \delta_{3} \delta_{2}\right)$.

In all cases, the Cayley graph $\mathcal{C}$ is $t$-transitive, for some $t \geq 2$ (and $t \leq 5)$.

If $\mathcal{P}$ is directly regular (chiral), then $\mathcal{C}$ is a 6 -fold cover of the 3 -transitive (2-transitive) graph $\mathcal{G}$. If $\mathcal{P}$ is non-orientably regular, then $\mathcal{C}$ is a 12 -fold cover of the 3 -transitive graph $\mathcal{G}$.

Proof. From [2, Ch. 18] we recall that a finite, symmetric trivalent graph like $\mathcal{C}$ is $t$-transitive for some $t$ satisfying $1 \leq t \leq 5$, and that the node stabilizer has order $3 \cdot 2^{t-1}$, which is at least $2 p=6$ for $\mathcal{C}$ (Proposition 11). Thus $t \geq 2$.

By [23, Thms. 2 and 5], $\mathcal{G}$ is 3-transitive (resp. 2-transitive) when $\mathcal{P}$ is regular (resp. chiral).

Remark. It is very unlikely that $\mathcal{C}$ could have transitivity $t=4$ or 5 , but we have no proof of this.

Example 16. The regular 4-simplex $\mathcal{P}=\{3,3,3\}$.

The automorphism group for this familiar self-dual, regular convex polytope is, of course, the Coxeter group $[3,3,3]$, so that $A \simeq S_{5}$ and $A^{+} \simeq A_{5}$. Recall that the longest element in $A$ is $\tau:=\sigma_{1} \sigma_{3} \sigma_{1} \sigma_{2}^{-1} \sigma_{1} \in A^{+}$. Then $\tau \delta$ is a central polarity in $D^{+}$, so that

$$
D^{+} \simeq A_{5} \times C_{2} .
$$

This group of order 120 is the icosahedral group [3,5]. With the new generators $\delta_{1}, \delta_{2}, \delta_{3}$ we get a Cayley graph $\mathcal{C}$ for the group [3,5], namely the 2-transitive graph 120B in [3]. Evidently 
we can think of $\delta_{1}, \delta_{2}, \delta_{3}$ as reflections in the sides of a triangular face of the icosahedron $\{3,5\}$.

The graph $\mathcal{C}$ is indeed a 6 -fold cover of the medial layer graph $\mathcal{G}$, which in turn is 3 transitive and is listed as 20B in [3]. We recall that $\mathcal{G}$ is the Levi graph for the Desargues configuration $10_{3}$.

Example 17. The 11 -cell $\mathcal{P}=\left\{\{3,5\}_{5},\{5,3\}_{5}\right\}$ and its relatives.

This very interesting polytope with 11 facets and (dually) 11 vertices, was independently discovered by Coxeter [8] and Grünbaum [13]. The enhanced Schläfli symbol indicates that $\mathcal{P}$ is the universal regular 4-polytope with hemi-icosahedral facets and hemi-dodecahedral vertex-figures. In fact, $\mathcal{P}$ is non-orientably regular, with $A \simeq P S L_{2}(11)$ of order 660 . Also, $G=D^{+} \simeq P G L_{2}(11)$.

The Cayley graph $\mathcal{C}$ is 2-transitive and has been described as graph $\mathrm{C} 1320.3$ in Conder's recently expanded census of symmetric trivalent graphs [4]. It is a 12 -fold cover of $\mathcal{G}$, which appears as the 3-transitive graph $\mathbf{1 1 0}$ in Foster's Census. (Here, and often elsewhere, our supporting calculations were done using GAP and the subsidiary packages GRAPE and nauty $[10,28,16]$.

For a somewhat different description of the 11-cell we refer to [21], where the polytope appears as a singular offshoot from a family of finite, self-dual (directly) regular polytopes $\mathcal{P}^{\pi}$, all with icosahedral facets and dodecahedral vertex-figures. The parameter $\pi$ is any prime in the ring $\mathbb{Z}[\tau]$ of algebraic integers in $\mathbb{Q}(\sqrt{5})$. In fact, the 11 -cell is a natural quotient of $\mathcal{P}^{\pi}$, when $\pi=-(2+5 \tau)$. On the other hand, if $\pi=2$, then $A \simeq O\left(4,2^{2},-1\right)$, the 4 dimensional orthogonal group over $G F\left(2^{2}\right)$ with Witt index 1 . The polytope $\mathcal{P}^{2}$ has 68 facets (and 68 vertices); its medial layer graph $\mathcal{G}$ must be $\mathrm{C} 1360.5$, the only 3 -transitive graph with 1360 nodes listed in [4].

Generally, if $\pi$ is an odd rational prime $\equiv \pm 2 \bmod 5$, then the number of nodes in $\mathcal{C}$ is about $\pi^{12}$.

Remark. Before proceeding, let us comment on the notation used in Example 17. A Petrie polygon in an abstract $n$-polytope $\mathcal{Q}$ is defined (inductively) as an edge-path along which any $n-1$ consecutive edges, but no $n$, belong to a Petrie polygon of a facet of $\mathcal{Q}$; the Petrie polygon of a polygon (2-polytope) is the polygon itself [17, p. 163].

For example, if $\mathcal{Q}$ is a regular polyhedron, say of type $\{p, q\}$, then all Petrie polygons have the same length $l$, being the period of $\rho_{0} \rho_{1} \rho_{2}$ in the automorphism group of $\mathcal{Q}$. If these data determine the combinatorial type of $\mathcal{Q}$, then we may denote $\mathcal{Q}$ by $\{p, q\}_{l}$. In particular, the hemi-icosahedron $\{3,5\}_{5}$ has pentagonal Petrie polygons, obtained by antipodal identification along the decagonal Petrie polygons of the icosahedron $\{3,5\}$.

In many cases, the regular polyhedron $\mathcal{Q}$ has a Petrie dual $\mathcal{Q}^{\pi}$, which shares the vertices and edges of $\mathcal{Q}$ but whose faces are the Petrie polygons of $\mathcal{Q}$. In particular, we have $\{p, q\}_{l}^{\pi} \simeq$ $\{l, q\}_{p}[17$, p. 192].

Example 18. The chiral polytopes $\{3,5,3\}_{l}$, for $l=7,9$.

In [9] we find a discussion of 'honeycombs' of type

$$
\{3,5,3\}_{l},
$$

for which the group $A^{+}$is obtained by adjoining the relation $\left(\sigma_{1} \sigma_{3}\right)^{l}=1$ to the standard relations listed in (2) (with $p=r=3, q=5$, of course). We recall that $l$ can be interpreted 
as the length of a 'right-handed' Petrie polygon; the 'left-handed' Petrie polygons have length $l^{\prime}$ equal the period of $\sigma_{2} \sigma_{1} \sigma_{2} \sigma_{3}$.

For $l \leq 6$ and $l=8$ the intersection condition (3) fails and the corresponding group is non-polytopal. (Even so, the resulting structures still have interesting medial layer graphs.) For $l \geq 10$, it seems, though we have no proof, that $A$ is infinite. Thus, we are left with $l=7$ or 9 , which somewhat surprisingly give finite, properly self-dual chiral polytopes.

The polytope $\{3,5,3\}_{9}$ has $l^{\prime}=10$ and group $A^{+} \simeq P S L(2,19)$, of order 3420 . The medial layer graph $\mathcal{G}$ appears as C1140.5 in [4]; and the Cayley graph $\mathcal{C}$ for $G=D^{+} \simeq$ $P G L(2,19)$ has 6840 nodes.

The polytope $\{3,5,3\}_{7}$ has $l^{\prime}=29$ and group $A^{+} \simeq P S L(2,29)$. However, this polytope admits a central polarity and so $D^{+} \simeq \operatorname{PSL}(2,29) \times C_{2}$.

Let us turn now to the case that $q$ is even. We recall from Lemma 8 that the index in $D^{+}$ of $G$ equals the index in $I_{2}(3)$ of the normal subgroup $f(\bar{K})$ generated by the images under (9) of all 'additional' relations. Various examples show that all possible quotients do occur: $D^{+} / G$ could be any one of $I_{2}(3), C_{2}$ or $\{1\}$. Therefore, we shall make do with organizing various subcases of interest.

Proposition 19. Suppose $\mathcal{P}$ is finite, self-dual and directly regular or chiral of type $\{3, q, 3\}$, with $q$ even. If $|G|=\left|D^{+}\right| / 6$, then $G$ acts regularly on the node set of $\mathcal{G}$ and $\mathcal{G} \simeq \mathcal{C}$.

Proof. This follows at once from Proposition 12 and the fact that both $\mathcal{G}$ and $\mathcal{C}$ have the same number of nodes.

Remark. We cannot take $\mathcal{P}$ to be non-orientably regular in Proposition 19. Indeed, from Proposition 12 we see that $\mathcal{C}$ is then at least a 2 -fold cover of $\mathcal{G}$.

Example 20. The 24-cell and other classical examples.

The medial layer graph for the 24-cell $\{3,4,3\}$ appears as the 3 -transitive graph 192A in [3]. Since $A^{+} \simeq[3,4,3]^{+}$is the rotation subgroup of a Coxeter group, there are no additional relations to adjoin to those in (2) (with $p=r=3, q=4$ ). Thus in Lemma 8 we have $f(\bar{K})=\{1\}$, so $k=1$ and $G$ has index 6 in $D^{+}$. By Proposition $19, \mathcal{G} \simeq \mathcal{C}$ really is a Cayley graph for the group $G$ of order 192 .

Now consider the hemi-24-cell $\{3,4,3\} / \pm 1$, which is also isomorphic to $\{3,4,3\}_{6}$, through imposition of the extra relation $\left(\sigma_{1} \sigma_{3}\right)^{6}=1$. Consulting (9), we still have $f(\bar{K})=$ $\{1\}$, so that $\mathcal{G} \simeq \mathcal{C}$ is the 3 -transitive graph 96 in [3].

The medial layer graph for the spherical polytope $\{3,2,3\}$ is $K_{3,3}$, which is indeed a Cayley graph for the group $I_{2}(3) \simeq S_{3}$.

Example 21. The universal locally toroidal polytopes

$$
\left\{\{3,6\}_{s},\{6,3\}_{s}\right\},
$$

for $s=(1,1),(2,0),(3,0)$.

We recall from $[17,1 \mathrm{D}]$ how the toroidal polyhedron $\{3,6\}_{s}$, with $s=(b, c)$, is obtained from $\mathcal{T}=\{3,6\}$, the regular tiling of the Euclidean plane by equilateral triangles. The rotation group for $\mathcal{T}$ is $[3,6]^{+}=\left\langle\sigma_{1}, \sigma_{2}\right\rangle$, whose defining relations are a suitable subset of (2):

$$
\sigma_{1}^{3}=\sigma_{2}^{6}=\left(\sigma_{1} \sigma_{2}\right)^{2}=1 \text {. }
$$


One obtains the toroid by identifying faces of $\mathcal{T}$ through the action of the normal subgroup of translations in $[3,6]^{+}$generated by $x^{b} y^{c}$, where $x:=\sigma_{2}^{2} \sigma_{1}^{-1}$ and $y:=\sigma_{2}^{-2} \sigma_{1}$ are unit translations along adjacent edges in the tiling. In brief, we adjoin to (11) the extra relation

$$
\left(\sigma_{2}^{2} \sigma_{1}^{-1}\right)^{b}\left(\sigma_{2}^{-2} \sigma_{1}\right)^{c}=1
$$

The resulting toroidal map is actually a 3 -polytope precisely when $b^{2}+b c+c^{2} \geq 3$ and is regular if and only if $b c(b-c)=0$. Otherwise, the polyhedron is chiral. We denote the dual of $\{3,6\}_{s}$ by $\{6,3\}_{s}$. Clearly this dual is obtained in like manner from the hexagonal tiling $\{6,3\}$. (Later we will need the remaining family of toroidal polyhedra $\{4,4\}_{s}$, for which $s=(b, c)$ satisfies $b^{2}+c^{2} \geq 2$.)

A polytope $\mathcal{P}$ is locally toroidal if its facets and vertex-figures are spherical or toroidal, with at least one kind toroidal. The regular locally toroidal polytopes have not yet been fully classified; see [17, Chs. 10-12].

Now let us consider the universal regular 4-polytope described by (10). This locally toroidal 4-polytope is known to be finite only when $s=(1,1),(2,0)$ or $(3,0)[17,11 \mathrm{E}]$. In each of these cases, the polytope is self-dual. Thus $D^{+}$is obtained from (2) and (5), with $p=r=3, q=6$, by adjoining just the relation (12); duality then forces the defining relation $\left(\sigma_{2}^{-2} \sigma_{3}\right)^{b}\left(\sigma_{2}^{2} \sigma_{3}^{-1}\right)^{c}=1$ for the vertex-figure.

From (12) and (9), we conclude that $f(\bar{K})$ is generated by

$$
\left(\alpha_{0}^{2} \alpha_{1} \alpha_{0}\right)^{b}\left(\alpha_{0}^{-2} \alpha_{0} \alpha_{1}\right)^{c}=\left(\alpha_{0} \alpha_{1}\right)^{c-b} \in I_{2}(3) .
$$

Thus it is trivial to apply Lemma 8. Drawing on [17, Table 11E1], we can summarize the results in the following table:

\begin{tabular}{c|c|c|c|c}
$s=(b, c)$ & $\left|D^{+}\right|$ & $k=|f(\bar{K})|$ & $|\mathcal{C}|$ & $|\mathcal{G}|$ \\
\hline$(1,1)$ & 108 & 1 & 18 & 18 \\
$(2,0)$ & 240 & 3 & 120 & 40 \\
$(3,0)$ & 2916 & 1 & 486 & 486 \\
\hline
\end{tabular}

For $s=(1,1)$, the medial layer graph $\mathcal{G} \simeq C$ is listed as $\mathbf{1 8}$ in [3] and, in fact, is the Levi graph for the Pappus configuration $9_{3}$. The corresponding group $G$ of order 18 is actually isomorphic to the rotation subgroup $[3,2,3]^{+}=\left\langle\eta_{1}, \eta_{2}, \eta_{3}\right\rangle$ for $\{3,2,3\}$, if identify $\delta_{1}$ with $\eta_{2}, \delta_{2}$ with $\eta_{1} \eta_{2} \eta_{3}$ and $\delta_{3}$ with $\eta_{1} \eta_{2}$.

When $s=(3,0)$, we again have $\mathcal{C} \simeq \mathcal{G}$, listed as the 3 -regular graph 486C in [3]. We note from [30] that the polytope $\mathcal{P}$ can also be described as $\{3,6,3\}_{6}$.

Finally, when $s=(2,0)$, we find that $\mathcal{C}$ is again the graph 120B in [3]; and once more $G$ is the icosahedral group $[3,5]$. However, now $\mathcal{C}$ is a 3 -fold cover of $\mathcal{G}$, which appears as $\mathbf{4 0}$ in [3].

Example 22. The universal polytope $\left\{\{3,2 b\}_{6},\{2 b, 3\}_{6}\right\}, b \geq 2$.

It is easy to check that

$$
\{6,3\}_{(b, 0)} \simeq\{6,3\}_{2 b}
$$


indicating that the combinatorial type of this toroidal polyhedron is forced by Petrie polygons having length $2 b$. Hence, for the dual of the Petrie dual, namely $\{3,2 b\}_{6}$, the crucial extra defining relation is

$$
\left(\rho_{0} \rho_{1} \rho_{2}\right)^{6}=\left(\sigma_{1}^{2} \sigma_{2}^{2}\right)^{3}=1 .
$$

The polyhedron $\{3,2 b\}_{6}$ has the same group of order $12 b^{2}$ as the original toroid (with different specified generators, of course).

In $[31, \S 5]$, the second author of this paper proved that the universal, self-dual regular polytope

$$
\left\{\{3,2 b\}_{6},\{2 b, 3\}_{6}\right\}
$$

has automorphism group of order

$$
|A|= \begin{cases}36 b^{4} & , b \text { odd } \\ 72 b^{4} & , b \text { even. }\end{cases}
$$

(We require $b \geq 2$ for the original toroidal map to be polyhedral.) From (14) and (9) we conclude that $f(\bar{K})$ is generated by $\left(\alpha_{0} \alpha_{1}\right)^{6}=1$, so that $k=1$ in all cases. Thus $\mathcal{G} \simeq \mathcal{C}$ is a 3 -transitive trivalent graph with $6 b^{4}$ (resp. $12 b^{4}$ ) nodes for $b$ odd (resp. even).

Example 23. Modular polytopes in the class $\left\langle\{3,6\}_{(b, c)},\{6,3\}_{(b, c)}\right\rangle$

We begin with the infinite Coxeter group $W=[3,6,3]$ and exploit its action on the conformal model of hyperbolic space $\mathbb{H}^{3}$. Referring to $[27, \S 10]$ or $[22, \S 6]$, we therefore consider the unimodular group $L_{2}^{\langle-1\rangle}(\mathbb{Z}[\omega])$, consisting of all $2 \times 2$ matrices of determinant \pm 1 over the domain of Eisenstein integers $\mathbb{Z}[\omega]$ (where $\omega=e^{2 \pi i / 3}$ ). Next let $H$ be the subgroup generated by

$$
S_{1}=\left[\begin{array}{rr}
1 & 1 \\
-1 & 0
\end{array}\right], S_{2}=\left[\begin{array}{rr}
\omega^{2} & 0 \\
-\omega^{2} & -\omega
\end{array}\right], S_{3}=\left[\begin{array}{rr}
\omega & 0 \\
0 & \omega^{2}
\end{array}\right],
$$

noting that $\operatorname{det}\left(S_{1}\right)=\operatorname{det}\left(S_{3}\right)=1, \operatorname{det}\left(S_{2}\right)=-1$ and $S_{1}^{3}=-I$ [22, Eq. 15]. We recall that $H$ has index 4 in $L_{2}^{\langle-1\rangle}(\mathbb{Z}[\omega])$ and that the rotation group

$$
W^{+}=[3,6,3]^{+} \simeq H /\{ \pm I\} .
$$

Of course, $[3,6,3]^{+}$is infinite, as is the corresponding polytope $\{3,6,3\}$, which is realized as a self-dual, regular tessellation of $\mathbb{H}^{3}$. However, if we reduce $H$ modulo $m \in \mathbb{Z}[\omega]$, then almost always we obtain a finite regular or chiral 4-polytope. More precisely, we first reduce $H$ to $H_{m}$, then examine

$$
X:=\left\{x \in \mathbb{Z}[\omega] /(m): x I \in H_{m}\right\},
$$

which is a group of units in $\mathbb{Z}[\omega] /(m)$ arising from the centre of $H_{m}$. For any admissible subgroup $S$ of such units, with $\{ \pm 1\} \subseteq S \subseteq X$, we set

$$
H_{m}^{S}:=H_{m} /\{s I: s \in S\}
$$

and let $\sigma_{j}$ be the natural image of $S_{j}$ in this quotient. By [22, Th. 6.1], if the non-zero modulus $m \nmid(1-\omega)$, then $A^{+}:=H_{m}^{S}=\left\langle\sigma_{1}, \sigma_{2}, \sigma_{3}\right\rangle$ is the rotation group for a finite 4-polytope $\mathcal{P}_{m}^{S}$. Briefly, $\mathcal{P}_{m}^{S}$ is directly regular if $m \mid \bar{m}$ and $S=\bar{S}$, but is chiral otherwise. 
For example, if $m=3$ then $\mathcal{P}_{3}^{\{ \pm 1\}}$ is the universal regular polytope

$$
\left\{\{3,6\}_{(3,0)},\{3,6\}_{(1,1)}\right\},
$$

which is clearly not self-dual. By [18, Th. 2], the medial layer graph $\mathcal{G}$ cannot then be symmetric. Indeed, $\mathcal{G}$ is the Gray graph, the smallest trivalent, semisymmetric graph [18, $\S 3.4]$.

In fact $\mathcal{P}_{m}^{S}$ is self-dual if and only if $\operatorname{gcd}(m, 1-\omega)=1$, which we now assume. We also suppose $m \nmid 2$, since $\mathcal{P}_{2}^{\{1\}} \simeq\{3,3,3\}$ collapses to the regular 4-simplex. Restricting $m=b-c w$ in this way (where $b, c \in \mathbb{Z}$ ), we have that $\mathcal{P}_{m}^{S}$ is a self-dual polytope in the class

$$
\left\langle\{3,6\}_{(b, c)},\{6,3\}_{(b, c)}\right\rangle .
$$

From [22] we note that

$$
\left|A^{+}\right|=\left|H_{m}^{S}\right|=\frac{2(m \bar{m})^{3}}{|S|} \prod_{\pi \mid m}\left(1-(\pi \bar{\pi})^{-2}\right),
$$

where the product is over all non-associated prime divisors $\pi$ of $m$ in $\mathbb{Z}[\omega]$. Thus we can readily compute the the number of nodes in the medial layer graph $\mathcal{G}$. Indeed, a typical node in $\mathcal{G}$ is the basic 2 -face $F_{2}$, whose stabilizer in $A^{+}$is the group of order 6 described in (7). Thus $\mathcal{G}$ has $\left|H_{m}^{S}\right| / 3$ nodes.

However, recalling our comments in Example 21, it is very unlikely that $\mathcal{P}_{m}^{S}$ will be universal for its facets and vertex-figures. Thus, in order to understand the corresponding Cayley graph $\mathcal{C}$, we must be a little more devious.

Proposition 24. For any modulus $m=b-c \omega \in \mathbb{Z}[\omega]$, with $\operatorname{gcd}(m, 1-\omega)=1$ and $m \neq 2$, and for any admissible unit group $S$, let $\mathcal{P}_{m}^{S}$ be the self-dual 4-polytope with rotation group $H_{m}^{S}$. Let $\mathcal{G}$ be its (trivalent) medial layer graph. Then

(a) if $m \mid \bar{m}$ and $S=\bar{S}$, then $\mathcal{P}_{m}^{S}$ is regular and $\mathcal{G}$ is 3-transitive.

(b) if $m \nmid \bar{m}$ or $S \neq \bar{S}$, then $\mathcal{P}_{m}^{S}$ is chiral and $\mathcal{G}$ is 2-transitive.

Furthermore, the associated (trivalent) Cayley graph $\mathcal{C}$ is transitive on 2 -arcs and is a $k$-fold cover of $\mathcal{G}$, where

$$
k:= \begin{cases}3, & \text { if } s^{2}=1 \text { for all } s \in S \\ 6, & \text { if } s^{2}=-1 \text { for some } s \in S\end{cases}
$$

Proof. The transitivity properties of $\mathcal{G}$ follow at once from [23, Ths. 2 and 5]. By Proposition 12 and Lemma 8 it remains only to compute $k=|f(\bar{K})|$. (Recall that $6=k\left[D^{+}: G\right]$ and that $\bar{K}$ has nothing to do with complex conjugation.)

From (13) we have $\left(\alpha_{1} \alpha_{0}\right)^{b-c} \in f(\bar{K}) \subseteq I_{2}(3)$. Now $m \bar{m}=b^{2}+b c+c^{2} \equiv(b-c)^{2}$ $(\bmod 3)$. Since, by hypothesis, $m$ is relatively prime to $3=-\omega^{2}(1-\omega)^{2}$, we conclude that $b-c$ is relatively prime to 3 , and so $\alpha_{0} \alpha_{1} \in f(\bar{K})$. Thus $k=3$ or 6 .

Now suppose $s^{2}=1$ for all $s \in S$. Thus, the determinant map is well-defined on $A^{+}=H_{m}^{S}$, and we have $\operatorname{det}\left(\sigma_{j}\right)=\operatorname{det}\left(\delta \sigma_{j} \delta\right)$ for $j=1,2,3$. With (6) this gives a welldefined homomorphism

$$
\begin{aligned}
h: D^{+} & \rightarrow\{ \pm 1\} \\
\alpha \delta^{i} & \mapsto \operatorname{det}(\alpha), \text { for } \alpha \in A^{+}, i=0,1 .
\end{aligned}
$$


Clearly $G \subseteq \operatorname{ker}(h)$, which has index 2 in $D^{+}$, since $\operatorname{det}\left(\sigma_{2}\right)=-1$. Thus $k=3$ in all such cases.

Otherwise we have $s^{2}=-1$ for some $s \in S$. But $s I=S_{i_{1}} S_{i_{2}} \cdots S_{i_{l}}$ for suitable $i_{j} \in\{1,2,3\}$. Since $\operatorname{det}\left(S_{l}\right)=-1$ only when $l=2$, we have $i_{j}=2$ for an odd number of $j$ 's. But in $H_{m}^{S}$ we have $1=\sigma_{i_{1}} \sigma_{i_{2}} \cdots \sigma_{i_{l}}$. From (9) we therefore conclude that $f(\bar{K})$ contains a reflection, so that $k=6$.

Remarks. Any rational prime $p \equiv 1(\bmod 3)$ factors over $\mathbb{Z}[\omega]$ as $p=\pi \bar{\pi}=b^{2}+$ $b c+c^{2}$, where $\pi=b-c \omega$ is prime in $\mathbb{Z}[\omega]$. Let us take our modulus $m$ to be $\pi$, with $S=\{ \pm 1\}$. We conclude from Proposition 24(b) that $\mathcal{P}_{\pi}^{\{ \pm 1\}}$ is self-dual and chiral of type $\left\{\{3,6\}_{(b, c)},\{6,3\}_{(b, c)}\right\}$. From (16) we have

$$
\left|A^{+}\right|=(\pi \bar{\pi})^{3}\left(1-(\pi \bar{\pi})^{-2}\right)=p\left(p^{2}-1\right) .
$$

Thus the medial layer graph $\mathcal{G}$ is 2 -transitive and has $p\left(p^{2}-1\right) / 3$ nodes. Since $k=3$, the Cayley graph $\mathcal{C}$ has $p\left(p^{2}-1\right)$ nodes and has an automorphism group which is transitive on 2 -arcs. For example, when $p=7$, we find that $\mathcal{G}$ and $\mathcal{C}$ are the 2 -transitive graphs $112 \mathrm{~A}$ and 336B, respectively, in [3].

Suppose now that the modulus $m$ is an odd rational prime $p$. For a moment we put aside the conformal action of $W^{+}$on $\mathbb{H}^{3}$ and turn instead to the standard faithful representation of $W=[3,6,3]$ as a crystallographic linear Coxeter group (see [15, Cor. 5.4] and [19, §4]). Abusing notation a little, we therefore now suppose $W \subset G L_{4}(\mathbb{Z})$, then reduce $W$ modulo $p$ and so obtain a finite representation $W_{p}$ in some orthogonal space $V$ over $\mathbb{Z}_{p}$. When $p>3$, the reduced group $W_{p}$ turns out to be the automorphism group of a finite, self-dual regular polytope $\mathcal{Q}_{p}$ in the class

$$
\left\langle\{3,6\}_{(p, 0)},\{6,3\}_{(p, 0)}\right\rangle \text {. }
$$

(See [20, Eqs. 22, 10]; this reference and [19] give a detailed description of the construction and applications to several natural familes of polytopes.)

We can now establish a connection with the earlier family of polytopes $\mathcal{P}_{m}^{S}$. For a suitable matrix $B \in G L_{4}(\mathbb{Q}(\omega))$, we may define an epimorphism

$$
\begin{aligned}
H & \rightarrow W^{+} \\
\text {with } A & \mapsto B(A \otimes \bar{A}) B^{-1} .
\end{aligned}
$$

As long as $s \bar{s}=1$ for all $s \in S$, this induces an epimorphism

$$
\lambda: H_{p}^{S} \rightarrow W_{p}^{+},
$$

which in fact becomes an isomorphism when $S$ is chosen carefully. We refer to $[19,20]$ for more notation and, without much explanation, summarize our calculations in

Corollary 25. For the rational prime $p>3$, let $\mathcal{Q}_{p}$ be the self-dual, directly regular 4 polytope with automorphism group $W_{p}=[3,6,3]_{p}$. Then the (trivalent) medial layer graph $\mathcal{G}$ for this polytope is 3 -transitive with $\left|W_{p}\right| / 6$ nodes. Furthermore, the associated (trivalent) Cayley graph $\mathcal{C}$ is transitive on 2-arcs and is a $k$-fold cover of $\mathcal{G}$, as indicated below:

\begin{tabular}{c|c|c|c|c}
$p(\bmod 12)$ & $S \subset \mathbb{Z}[\omega] /(p)$ & $H_{p}^{S} \simeq W_{p}^{+} \simeq$ & $\left|W_{p}\right|$ & $k$ \\
\hline 1 & $\{ \pm 1, \pm s\}$ & $O_{1}(4, p, 1)$ & $p^{2}\left(p^{2}-1\right)^{2}$ & 6 \\
\hline 5 & $\{ \pm 1\}$ & $O(4, p,-1)$ & $2 p^{2}\left(p^{4}-1\right)$ & 3 \\
\hline 7 & $\{ \pm 1\}$ & $O(4, p, 1)$ & $2 p^{2}\left(p^{2}-1\right)^{2}$ & 3 \\
\hline 11 & $\{ \pm 1, \pm s\}$ & $O_{1}(4, p,-1)$ & $p^{2}\left(p^{4}-1\right)$ & 6 \\
\hline
\end{tabular}


Proof. For $p \equiv \pm 1(\bmod 12)$ there exists $s \in \mathbb{Z}[\omega]$ with $s^{2} \equiv-1, s \bar{s} \equiv 1(\bmod p)$. By Proposition 24 we then have $k=6$.

Note that $\mathcal{C}$ has $p^{2}\left(p^{2}-\epsilon\right)\left(p^{2}-1\right)$ nodes, where $\epsilon=+1$ for $p \equiv 1,7(\bmod 12), \epsilon=-1$ for $p \equiv 5,11(\bmod 12)$. For example, when $p=5$, the graph $\mathcal{C}$ with 15600 nodes is actually 2 -transitive and is a 3 -fold cover of the 3 -transitive graph $\mathcal{G}$.

\section{Finite polytopes $\mathcal{P}$ of type $\{4, q, 4\}$}

Suppose $\mathcal{P}$ is a finite, self-dual and regular or chiral polytope of type $\{4, q, 4\}$. Then our observations in Section 3 tell us that the corresponding medial layer graph $\mathcal{G}$ and Cayley graph $\mathcal{C}$ are symmetric tetravalent graphs. The general theory of these graphs is somewhat less developed than in the trivalent case, although considerable information can be found in [2, Ch. 17] and in [11, 24, 32]. We shall have correspondingly less to say here about such graphs as arise from polytopes. We summarize what we can easily say in the following

Proposition 26. Suppose $\mathcal{P}$ is finite, self-dual and regular or chiral of type $\{4, q, 4\}$. The corresponding Cayley graph $\mathcal{C}$ and medial layer graph $\mathcal{G}$ are symmetric, tetravalent graphs. Furthermore, $\mathcal{C}$ is a l-fold cover of $\mathcal{G}$, as described in the following Table:

\begin{tabular}{|c|c|c|c|}
\hline $\mathcal{P}$ & $|\mathcal{G}|$ & $q$ odd & $q$ even \\
\cline { 3 - 4 } & & $l=$ & $l=$ \\
\hline directly regular & $\left|D^{+}\right| / 8$ & 8 or 4 & $8,4,2$ or 1 \\
\hline chiral & $\left|D^{+}\right| / 8$ & 8 or 4 & $8,4,2$ or 1 \\
\hline non-orientably regular & $\left|D^{+}\right| / 16$ & 16 or 8 & $16,8,4$ or 2 \\
\hline
\end{tabular}

In particular, if $\mathcal{P}$ is directly regular or chiral and $|G|=\left|D^{+}\right| / 8$, then $\mathcal{C} \simeq \mathcal{G}$. This isomorphism is impossible when $q$ is odd and in all non-orientably regular cases.

Proof. This follows immediately from Propositions 9, 11 and 12.

Example 27. The cubic toroids $\{4,3,4\}_{s}$, where $s=(b, 0,0),(b, b, 0)$ or $(b, b, b)$, for integers $b \geq 2$.

The cubic toroid $\mathcal{P}_{s}:=\{4,3,4\}_{s}$ is a finite, self-dual and directly regular 4-polytope constructed in much the same way as the toroidal polyhedra described in Example 21. Thus $\mathcal{P}_{s}$ is a quotient of $\{4,3,4\}$, the familiar tessellation of Euclidean 3 -space by cubes; hence, we can regard $\mathcal{P}_{s}$ as a tessellation of the 3 -torus [17, 6D].

The toroids $\mathcal{P}_{s}$ are parametrized by a type vector $s=\left(b^{i}, 0^{3-i}\right)$, as indicated above. From $[17,6 \mathrm{D} 5]$ we find that the group $A^{+}$is defined by the relations in (2), with $p=r=4, q=3$, along with a single extra relation

$$
\left(\sigma_{1} \sigma_{3} \beta\right)^{b}=1
$$

where $\beta=\sigma_{2}^{2}, \sigma_{1} \sigma_{3} \sigma_{2}$ or $\left(\sigma_{1} \sigma_{3}\right)^{2}$, for $s=(b, 0,0),(b, b, 0)$ or $(b, b, b)$, respectively. Noting that $\sigma_{2}^{3}=1$, it is now easy to use (9) and Lemma 8 to show that the covering multiplicity $k=f(\bar{K})=4$ in every case. Drawing on [17, table 6D1], we summarize our calculations here: 


\begin{tabular}{|c|c|c|c|c|}
\hline$s$ & $|A|$ & $|\mathcal{G}|=|A| / 8$ & $k$ & $|\mathcal{C}|$ \\
\hline$(b, 0,0)$ & $48 b^{3}$ & $6 b^{3}$ & 4 & $24 b^{3}$ \\
\hline$(b, b, 0)$ & $96 b^{3}$ & $12 b^{3}$ & 4 & $48 b^{3}$ \\
\hline$(b, b, b)$ & $192 b^{3}$ & $24 b^{3}$ & 4 & $96 b^{3}$ \\
\hline
\end{tabular}

The smallest toroid in this class is in some sense the most interesting. The polytope $\mathcal{P}_{(2,0,0)}$ is flat, meaning that each of the 8 vertices is incident with each of the 8 facets. Of course, the 24 edges and 24 polygons comprise the 48 nodes of the medial layer graph $\mathcal{G}$, whose automorphism group is just the full duality group $D$ of order 768 . This graph is listed as $C 4[48,10]$ in [32] and is 1-transitive.

The Cayley graph $\mathcal{C}$ has $192=4 \cdot 48$ vertices; and $\operatorname{Aut}(\mathcal{C})$ has the unexpectedly large order $98304=192 \cdot 4 \cdot 2^{7}$. From [2, p. 134], we conclude that $\mathcal{C}$ is 1 -transitive, and that the stabilizer of a 1 -arc has order $2^{7}$.

Example 28. The universal polytope $\left\{\{4,2 b\}_{4},\{2 b, 4\}_{4}\right\}, b \geq 2$.

This family of finite, self-dual regular polytopes is also described in $[31, \S 5]$. The analysis is very similar to that in Example 22, so we merely record some results. The toroidal map

$$
\{4,4\}_{(b, b)} \simeq\{4,4\}_{2 b}
$$

has automorphism group of order $16 b^{2}$. The dual of the Petrie dual is $\{4,2 b\}_{4}$ and the selfdual, universal regular polytope $\left\{\{4,2 b\}_{4},\{2 b, 4\}_{4}\right\}$ has $|A|=64 b^{4}$. Again we have $k=1$ in all cases, so that $\mathcal{G} \simeq \mathcal{C}$ is a tetravalent graph on $8 b^{4}$ nodes, with group transitive on 1-arcs.

Example 29. Modular polytopes in the class $\left\langle\{4,4\}_{(p, 0)},\{4,4\}_{(p, 0)}\right\rangle$, for odd primes $p$.

The infinite Coxeter group $W=[4,4,4]$ is crystallographic. If, as in the previous section, we reduce the standard linear representation modulo any odd prime $p$, we find that $A=W_{p}$ is the automorphism group of a finite, self-dual and directly regular polytope $\mathcal{P}_{p}$ in the class $\left\langle\{4,4\}_{(p, 0)},\{4,4\}_{(p, 0)}\right\rangle[20$, pp. 340-343]. This locally toroidal polytope is universal for its class when $p=3$, but almost surely is not for larger primes [17, 10C]. Consulting [20, Eq. (6)], we note that

$$
A=W_{p}=\left\{\begin{array}{lll}
O_{1}(4, p, 1), & \text { if } p \equiv 1 & (\bmod 8) \\
O_{1}(4, p,-1), & \text { if } p \equiv 7 & (\bmod 8) \\
O(4, p, 1), & \text { if } p \equiv 5 & (\bmod 8) \\
O(4, p,-1), & \text { if } p \equiv 3 & (\bmod 8)
\end{array}\right.
$$

From here on the analysis is very similar to that for Corollary 25. We thus obtain

Corollary 30. For any odd prime $p$, let $\mathcal{P}_{p}$ be the self-dual, directly regular 4-polytope with automorphism group $W_{p}=[4,4,4]_{p}$. Then the symmetric tetravalent Cayley graph $\mathcal{C}$ is a $k$-fold cover of the symmetric, tetravalent medial layer graph $\mathcal{G}$, where

$$
k:=\left\{\begin{array}{lll}
8 & \text { if } p \equiv \pm 1 \quad(\bmod 8) \\
4 & \text { if } p \equiv \pm 3 \quad(\bmod 8)
\end{array} .\right.
$$

We conclude this section with a look at

Example 31. The universal, locally projective polytope $\left\{\{4,3\}_{3},\{3,4\}_{3}\right\}$ [25, Table 1]. 
The facets of this self-dual, non-orientably regular polytope are hemicubes; dually its vertex-figures are hemi-octahedra. Along with the standard relations in (2) and (5), with $p=r=4, q=3$, we need only enforce $\left(\rho_{0} \rho_{1} \rho_{2}\right)^{3}=1$, or equivalently

$$
\left(\sigma_{2} \sigma_{1}^{2}\right)^{3}=1 \text {. }
$$

This gives $|A|=\left|A^{+}\right|=96$. From (9), we conclude that $f(\bar{K})$ is the normal subgroup generated by $\left(\alpha_{0}\left(\alpha_{0} \alpha_{1}\right)^{2}\right)^{3}=\alpha_{1} \alpha_{0} \alpha_{1}$ in $I_{2}(4)$, so that $k=4$.

Note that $\mathcal{G}$ has 12 nodes. From Proposition 12, we find that $\mathcal{C}$ has 96 nodes and is a 8 -fold cover of $\mathcal{G}$. Both $\mathcal{C}$ and $\mathcal{G}$ are 1 -transitive.

\section{Other examples and questions}

We end our survey of particular examples with a look at

Example 32. The classical 4-dimensional star-polytopes.

Consulting $[17,7 \mathrm{D}]$, we find that just three of the 4-dimensional regular star-polytopes concern us here: $\left\{5, \frac{5}{2}, 5\right\},\left\{\frac{5}{2}, 5, \frac{5}{2}\right\}$ and $\left\{5,3, \frac{5}{2}\right\}$.

The first two of these are geometrically self-dual, and, as abstract regular polytopes, are each isomorphic to the universal polytope

$$
\{\{5,5 \mid 3\},\{5,5 \mid 3\}\}
$$

([17, Th. 7D16(b)]). The notation indicates, for instance, that the facet $\{5,5 \mid 3\}$, with Schläfli type $\{5,5\}$, is specified by its having triangular holes. This means that $\left(\sigma_{1} \sigma_{2}^{-1}\right)^{3}=$ 1. Of course, the full automorphism group $A$ is the Coxeter group $H_{4}$ of order 14400 , so that $\mathcal{G}$ is a symmetric pentavalent graph with 1440 nodes. By Propositions 9 and 12, we see that $\mathcal{C}$ is a symmetric pentavalent graph on 14400 nodes and is a 10 -fold cover of $\mathcal{G}$.

Now although the other polytope $\left\{5,3, \frac{5}{2}\right\}$ is clearly not geometrically self-dual, it is nevertheless self-dual in the combinatorial sense used throughout this paper. In fact, it is isomorphic to

$$
\{5,3,5 \mid 3\}
$$

whose deep triangular holes are specified by $\left(\sigma_{1} \sigma_{3} \sigma_{2}^{-1}\right)^{3}=1$ [17, Th. 7D16(c)]. We obtain new pentavalent graphs $\mathcal{G}$ and $\mathcal{C}$, again with 1440 and 14400 nodes, respectively.

We conclude by mentioning some open questions. First of all, suppose $\mathcal{G}$ is $t$-transitive, while the cover $\mathcal{C}$ is $s$-transitive. Invariably we have observed that $s \leq t$; however, we have no proof that this must be so. Of course, it would be very desirable to find examples with say $s=4$ or 5 in the trivalent case (where $t=2$ or 3 ); but this is most unlikely.

In Proposition 14, we have described $\mathcal{C}$ as a derived voltage graph. If also $A$ acts as a group of automorphisms on the stabilizer $S$, in a manner compatible with the voltage assignment $\varphi$, it is at least possible to lift $t$-transitivity on $\mathcal{G}$ to $\mathcal{C}$, so that $s \geq t$ [2, Prop. 19.4]. However, we have no non-trivial examples of this very special situation. Also, we do not know if the voltage graph construction in Proposition 14 can be extended in a natural way to non-orientably regular cases (but see [12, Th. 2.2.2]).

Finally, we reconsider improperly self-dual chiral polytopes $\mathcal{P}$ of type $\{p, q, p\}$. If $q$ is even, then $\mathcal{P}$ might not admit a polarity (duality of period 2); but when $q$ is odd, $\mathcal{P}$ does admit polarities [14, Th. 3.4]. Nevertheless, we have avoided all such polytopes in order to simplify the definition of the graph $\mathcal{C}$. Perhaps these complications can be overcome to yield interesting results in improperly self-dual cases. 


\section{References}

[1] N. Biggs, Presentations for Cubic Graphs, in: Michael D. Atkinson (ed.), Computational Group Theory, Academic Press, London, 1984, 57-63.

[2] N. Biggs, Algebraic Graph Theory, second ed., Cambridge University Press, Cambridge, 1993.

[3] I. Z. Bouwer, W. W. Chernoff, B. Monson and Z. Star (eds.), The Foster Census: R.M. Foster's Census of Connected Symmetric Trivalent Graphs, The Charles Babbage Research Centre, Winnipeg, 1988.

[4] M. Conder, Trivalent (cubic) symmetric graphs: http://www. math. auckland.ac.nz/ conder/symmcubic2048list.txt.

[5] M. Conder and P. Lorimer, Automorphism Groups of Symmetric Graphs of Valency 3, Journal of Combinatorial Theory, Series B 47 (1989), 60-72.

[6] M. Conder and P. Dobcsányi, Trivalent symmetric graphs on up to 768 vertices, J. Combinatorial Mathematics and Combinatorial Computing 40 (2002), 41-63.

[7] H.S.M. Coxeter, The edges and faces of a 4-dimensional polytope, Congressus Numerantium 28 (1980), 309-334.

[8] H.S.M. Coxeter, A symmetrical arrangement of eleven hemi-icosahedra. in: M. Rosenfeld and J. Zaks (eds.), Convexity and Graph Theory, Jerusalem, 1981, North-Holland Math. Stud. 87, North-Holland, Amsterdam, 1984, 103-114.

[9] H. S. M. Coxeter and A. I. Weiss, Twisted honeycombs $\{3,5,3\}_{t}$ and their groups, Geom. Dedicata 17 (1984), 169-179.

[10] The GAP Group, GAP - Groups, Algorithms, and Programming, Version 4.3 (2002):

http://www.gap-system.org.

[11] A. Gardiner and C. Praeger, On 4-valent symmetric graphs, Europ. J. Combinatorics 15 (1994), 375-381.

[12] J. L. Gross and T. W. Tucker, Topological Graph Theory, Dover, New York, 2001.

[13] B. Grünbaum, Regularity of graphs, complexes and designs, Problèmes combinatoires et théorie des graphes, Colloq. Internat. C.N.R.S. No. 260, Orsay, 1977, 191-197.

[14] I. Hubard and A. I. Weiss, Self-duality of Chiral Polytopes, Journal of Combinatorial Theory, Series A 111 (2005), 128-136.

[15] J. E. Humphreys, Reflection Groups and Coxeter Groups, Cambridge Univ. Press, Cambridge, 1990.

[16] B.D. McKay, The nauty package:

http://cs.anu.edu.au/people/bdm/nauty/.

[17] P. McMullen and E. Schulte, Abstract Regular Polytopes, Encyclopedia of Mathematics and its Applications 92, Cambridge University Press, Cambridge, 2002.

[18] B. Monson, T. Pisanski, E. Schulte and A. I. Weiss, Semisymmetric Graphs from Polytopes, Journal of Combinatorial Theory, Series A 114 (2007), 421-435.

[19] B. Monson and E. Schulte, Reflection Groups and Polytopes over Finite Fields - I, Advances in Applied Mathematics 33 (2004), 290-317.

[20] B. Monson and E. Schulte, Reflection Groups and Polytopes over Finite Fields - II, Advances in Applied Mathematics 38 (2007), 327-356.

[21] B. Monson and E. Schulte, Modular Reduction in Abstract Polytopes, Canadian Mathematical Bulletin, to appear. 
[22] B. Monson and A. I. Weiss, Eisenstein Integers and Related C-groups, Geometriae Dedicata 66 (1997), 99-117.

[23] B. Monson and A. I. Weiss, Medial Layer Graphs of Equivelar 4-polytopes, European Journal of Combinatorics 28 (2007), 43-60.

[24] P. Potočnik and S. Wilson, Tetravalent edge-transitive graphs of girth at most 4, Journal of Combinatorial Theory, Series B 97 (2007), 217-236.

[25] E. Schulte, Amalgamation of Regular Incidence-Polytopes, Proc. London Math. Soc. 56 (1988), 303-328.

[26] E. Schulte and A. I. Weiss, Chiral polytopes, in: P. Gritzmann and B. Sturmfels (eds.), Applied Geometry and Discrete Mathematics. The Victor Klee Festschrift, DIMACS Series in Discrete Mathematics and Theoretical Computer Science 4, AMS, Providence, 1991, 493-516.

[27] E. Schulte and A. I. Weiss, Chirality and projective linear groups, Discrete Mathematics 131 (1994), 221-261.

[28] L. H. Soicher, The GRAPE package for GAP:

http://www.maths.qmul.ac.uk/ leonard/grape/.

[29] A. I. Weiss, On trivalent graphs embedded in twisted honeycombs, in: A. Barlotti, P. V. Ceccherini and G. Tallini (eds.), Combinatorics '81 (Rome, 1981), North-Holland Math. Stud. 78, NorthHolland, Amsterdam, 1983, 781-787.

[30] A. I. Weiss, An infinite graph of girth 12, Trans. Amer. Math. Soc. 283 (1984), 575-588.

[31] A. I. Weiss, Some infinite families of finite incidence-polytopes, Journal of Combinatorial Theory, Series A 55 (1990), 60-73.

[32] S. Wilson and P. Potočnik, A census of edge-transitive tetravalent graphs:

http://jan.ucc.nau.edu/ swilson/C4Site/. 